\title{
Polarization-Dependent SFG Spectroscopy of Near Ambient Pressure CO Adsorption on Pt(111) and Pd(111) Revisited
}

\author{
$\mathrm{Xia} \mathrm{Li}^{1}$ · Matteo Roiaz ${ }^{1}$. Verena Pramhaas ${ }^{1}$. Christoph Rameshan ${ }^{1} \cdot$ Günther Rupprechter ${ }^{1}$
}

Published online: 13 April 2018

(c) The Author(s) 2018

\begin{abstract}
Polarization-dependent sum frequency generation (SFG) vibrational spectroscopy was employed to examine CO overlayers on $\mathrm{Pt}(111)$ and $\mathrm{Pd}(111)$ single crystal surfaces at room temperature. Utilizing different polarization combinations (SSP and PPP) of the visible and SFG light allows to determine the molecular orientation (tilt angle) of interface molecules but the analysis of the measured $I_{\mathrm{ppp}} / I_{\mathrm{ssp}}$ is involved and requires a proper optical interface model. For $\mathrm{CO} / \mathrm{Pt}(111)$, the hyperpolarizability ratio $\left(R=\beta_{a a c} / \beta_{c c c}=\beta_{b b c} / \beta_{c c c}\right)$ is not exactly known and varying $R$ in the range $0.1-0.5$ yields tilt angles of $40^{\circ}-0^{\circ}$, respectively. Based on the known perpendicular adsorption of $\mathrm{CO}$ on $\mathrm{Pt}$, an exact $R$-value of 0.49 was determined. Polarization-dependent SFG spectra in the pressure range $10^{-4}$ to $36 \mathrm{mbar}$ did not indicate any change of the tilt angle of adsorbed CO. Modeling also indicated a strong dependence of $I_{\mathrm{ppp}} / I_{\mathrm{ssp}}$ on the incidence angles of visible and IR laser beams. Complementing previous low temperature/low pressure data, room temperature $\mathrm{CO}$ adsorption on $\mathrm{Pd}(111)$ was examined from $10^{-6}$ to 250 mbar. The absolute PPP and SSP spectral intensities on Pt and Pd were simulated, as well as the expected $I_{\mathrm{ppp}} / I_{\mathrm{ssp}}$ ratios. Although $\mathrm{CO}$ on Pt and Pd should exhibit similar intensities (at high CO coverage), the higher $I_{\mathrm{ppp}} / I_{\mathrm{ssp}}$ ratio for Pd (48 vs. 27 on Pt) renders the detection of adsorbed CO in SSP spectra difficult. The presence or absence of CO species in SSP spectra can thus not simply be correlated to tilted or perpendicular CO molecules, respectively. Careful modeling, including not only molecular and interface properties, but also the experimental configuration (incidence angles), is certainly required even for seemingly simple adsorbate-substrate systems.
\end{abstract}

Keywords Sum frequency generation $\cdot$ Carbon monoxide $\cdot$ Single crystals $\cdot$ Orientation $\cdot$ Model catalysis

\section{Introduction}

For many years, the surface science approach to heterogeneous catalysis was restricted to gas pressures of $10^{-6}$ mbar and below, giving rise to the well-known "pressure gap" problem [1-3]. This limitation was overcome when surface-sensitive methods (or modes thereof) were developed that could be operated at least in the mbar pressure range. Among the first were sum frequency generation (SFG) vibrational spectroscopy and high pressure scanning tunneling microscopy (HP-STM), both methods and their application to catalytic problems being pioneered by Somorjai and coworkers [4-6]. A specific asset of SFG was the ability to monitor adsorbed molecules in situ during the

Günther Rupprechter

guenther.rupprechter@tuwien.ac.at

1 Institute of Materials Chemistry, TU Wien, 1060 Vienna, Austria ongoing catalytic reaction at mbar gas pressure and elevated temperature, i.e. under technologically relevant conditions [7]. To date, Somorjai's group has examined a vast range of reaction systems, mostly on Pt surfaces, spanning from ethylene, propylene and cyclohexene hydrogenation/dehydrogenation via $\mathrm{CO}$ oxidation/dissociation and $\mathrm{CO} /$ hydrocarbon coadsorption to selective aldehyde hydrogenation [8-13], to name just a few. As Pt model catalysts, various low-Millerindex as well as stepped and kinked single crystal surfaces were utilized (linking the SFG in situ work to Somorjai's early high pressure cell studies without spectroscopy), complemented by thin films as supports for shape-controlled colloidal nanoparticles $[14,15]$. This enabled to examine metal-support interactions for nanoparticles that often occur in reducing (hydrogen) atmosphere [16-18].

SFG vibrational spectroscopy can also be used to probe the orientation (tilt angle) of molecules adsorbed on metallic, semiconductor and insulator surfaces, making use of the dependence of intensities of different polarization 
combinations on the orientation of the surface molecule [19-23]. In this context, the molecular adsorbate structures of $\mathrm{CO}$ on $\mathrm{Pt}(111)[23,24]$ and $\mathrm{Pd}(111)$ [22] have been studied, but results on the orientation (tilt angle) of $\mathrm{CO}$ on single-crystal surfaces were somewhat ambiguous. This is due to the complexity of the orientation analysis, which is based on polarization-dependent SFG spectroscopy, and the analysis/modeling of the observed intensities (which sometimes includes simplifications and/or assumptions that may not be justified). In this contribution, we revisit the benchmark systems of $\mathrm{CO} / \mathrm{Pt}(111)$ and $\mathrm{CO} / \mathrm{Pd}(111)$, employing a new SFG setup (UHV to mbar) [25] and specifically discuss the orientation analysis in detail, also reflecting analogies and differences to previous studies.

\section{Basic Theory of SFG}

SFG is a second-order nonlinear optical process during which two photons of certain frequencies interact simultaneously with a surface molecule to instantaneously emit a new photon at the sum of the two frequencies. The unique advantage of SFG, distinct from other surface-sensitive techniques, is attributed to its interface selectivity. This originates from the fact that coherent second-order optical processes are forbidden in media with inversion symmetry, while they are allowed for an interface layer, where this centrosymmetry is naturally broken. The SFG intensity $\left(I_{S F G}\right)$ is proportional to the two incident laser intensities $\left(I_{V i s}\right.$ and $I_{I R}$ ) and the absolute square of second-order nonlinear susceptibility $\left(\chi_{\text {eff }}^{(2)}\right)$, as shown in Eq. 1.

$I_{S F G} \propto\left|\chi_{\mathrm{eff}}^{(2)}\right|^{2} I_{V i S} I_{I R}$

The surface susceptibility $\chi_{\text {eff }}^{(2)}$ is composed of a non-resonant $\left(\chi_{N R}^{(2)}\right)$ and resonant part $\left(\chi_{R}^{(2)}\right)$

$\chi_{\mathrm{eff}}^{(2)}=\chi_{N R}^{(2)}+\chi_{R}^{(2)}=\chi_{0} e^{i \phi}+\sum \frac{\chi_{q}}{\omega_{I R}-\omega_{q}+i \Gamma_{q}}$

$\chi_{0}$ is the magnitude of the non-resonant susceptibility $\chi_{N R}^{(2)}$ due to electronic excitations of the substrate and the adsorbate, and $\phi$ is its phase relative to the resonant term. $\chi_{q}, \omega_{q}$ and $\Gamma_{q}$ represent the resonance amplitude, frequency and damping constant of the $q$ th vibrational mode, respectively. $\omega_{I R}$ is the frequency of the IR laser beam. In general, $\chi_{N R}^{(2)}$ should be small and real when the substrate is not resonant with either $\omega_{i}$ of incident visible, IR and output SFG beams. For dielectric interfaces, it is negligible. However, for metal or semiconductor substrate interfaces, $\chi_{N R}^{(2)}$ generally becomes complex and can no longer be ignored. $\chi_{\text {eff }}^{(2)}$ depends on the experimental polarization and geometry, and there are an infinite number of combinations of experimental configurations that can give different $\chi_{\text {eff }}^{(2)}$. In this paper, we mainly study $\chi_{\text {eff }}^{(2)}$ with a linear combination of independent experimental polarization combinations, namely, SSP (S-polarized sum frequency, S-polarized visible and P-polarized infrared) and PPP as shown in the following:

$$
\begin{gathered}
\chi_{\mathrm{eff}, S S P}^{(2)}=L_{y y}\left(\omega_{S F G}\right) L_{y y}\left(\omega_{V i s}\right) L_{z z}\left(\omega_{I R}\right) \sin \alpha_{I R} \chi_{y y z} \\
\chi_{\mathrm{eff}, P P P}^{(2)}=-L_{x x}\left(\omega_{S F G}\right) L_{x x}\left(\omega_{V i s}\right) L_{z z}\left(\omega_{I R}\right) \cos \alpha_{S F G} \cos \alpha_{V i s} \sin \alpha_{I R} \chi_{x x z} \\
-L_{x x}\left(\omega_{S F G}\right) L_{z z}\left(\omega_{V i s}\right) L_{x x}\left(\omega_{I R}\right) \cos \alpha_{S F G} \sin \alpha_{V i s} \cos \alpha_{I R} \chi_{x z x} \\
+L_{z z}\left(\omega_{S F G}\right) L_{x x}\left(\omega_{V i s}\right) L_{x x}\left(\omega_{I R}\right) \sin \alpha_{S F G} \cos \alpha_{V i s} \cos \alpha_{I R} \chi_{z x x} \\
+L_{z z}\left(\omega_{S F G}\right) L_{z z}\left(\omega_{V i s}\right) L_{z z}\left(\omega_{I R}\right) \sin \alpha_{S F G} \sin \alpha_{V i s} \sin \alpha_{I R} \chi_{z z z}
\end{gathered}
$$

Here $\omega_{S F G}, \omega_{V i s}$ and $\omega_{I R}$ are the frequencies; $\alpha_{S F G}, \alpha_{V i s}$ and $\alpha_{I R}$ are the angles (with respect to the surface normal), of the SFG signal, visible and IR laser beams, respectively. $L_{i i}\left(\omega_{i}\right)$ denotes the Fresnel factor at frequency $\omega_{i}$ for the local field corrections, which can be calculated with the knowledge of $\alpha_{i}$ and refractive indices $n_{1}$ (medium 1, the air in which incident and SFG photons propagate), $n_{2}$ (medium 2 , the single crystal phase) and $n^{\prime}$ (interfacial layer). In this paper, the $n^{\prime}$ values were estimated by the modified Lorentz model, and the expression is $n^{\prime}=n_{1} n_{2} \sqrt{\frac{6+n_{2}^{2}-n_{1}^{2}}{4 n_{2}^{2}+2 n_{1}^{2}}}$ [26] ; when $n_{1}=1$, then $n^{\prime}=n_{2} \sqrt{\frac{n_{2}{ }^{2}+5}{4 n_{2}{ }^{2}+2}}$ [27]. $\chi_{i j k}^{(2)}$ is the macroscopic sum frequency susceptibility, which is related to the microscopic hyperpolarizability tensor elements $\beta_{i^{\prime} j^{\prime} k^{\prime}}^{(2)}$ in the molecular coordinates system. $\chi_{i j k}^{(2)}$ can be deduced from SFG measurement with three different input/output polarization combinations, for example, SSP, SPS, PSS and PPP.

For $C_{\infty v v}$ symmetry group, such as $\mathrm{CO}, \mathrm{OH},-\mathrm{CH}$, there are only two independent nonvanishing components in $\beta_{i^{\prime} j^{\prime} k^{\prime}}^{(2)}, \beta_{c c c}$ and $\beta_{a a c}=\beta_{b b c}$. Then, the non-zero macroscopic elements of $\chi_{i j k}^{(2)}$ for a rotationally isotropic interface which are obtained through integration over the Euler angles $(\phi$, azimuth angle; $\psi$, twist angle) can be expressed as follows $[27,28]$.

$\chi_{x x z}^{(2)}=\chi_{y y z}^{(2)}=\frac{1}{2} N_{s} \beta_{c c c}\left[(1+R)\langle\cos \theta\rangle-(1-R)\langle\cos \theta\rangle^{3}\right]$

$\chi_{x z x}^{(2)}=\chi_{z x x}^{(2)}=\chi_{y z y}^{(2)}=\chi_{z y y}^{(2)}=\frac{1}{2} N_{s} \beta_{c c c}\left[(1-R)\langle\cos \theta\rangle-(1-R)\langle\cos \theta\rangle^{3}\right]$ $\chi_{z z z}^{(2)}=N_{s} \beta_{c c c}\left[R\langle\cos \theta\rangle+(1-R)\langle\cos \theta\rangle^{3}\right]$

Here $\beta_{i^{\prime} j^{\prime} k^{\prime}}^{(2)}$ is the molecular hyperpolarizability tensor. The hyperpolarizability ratio is $R=\beta_{a a c} / \beta_{c c c}=\beta_{b b c} / \beta_{c c c}$. For a single bond with $C_{\infty v v}$ symmetry, the $R$-value equals to the bond polarizability derivative ratio $r$. Different $R$-values will 
lead to different results in the SFG orientational analysis [21, $27,28] . \theta$ is the orientation angle of the moiety of the symmetry axis with respect to the surface normal. $N_{s}$ is the effective surface number density of molecules contributing to the SF signal. Then, based on Eqs. 1-4, we can determine the orientation $(\theta)$ and/or molecular hyperpolarizability ratio $(R)$ of the moiety by the measurements of the ratio of independent nonvanishing $\chi_{i j k}^{(2)}$ components assuming a $\delta$-function distribution for $\theta$. The latter assumption is frequently applied because only a small angular distribution is expected.

\section{Experimental Section}

The experiments on $\operatorname{Pt}(111)$ and $\operatorname{Pd}(111)$ single crystals were performed in a new UHV surface analysis system equipped with an SFG-compatible UHV-high pressure cell [25] (setup similar to that described in Refs. [2, 29]). Both Pt and Pd surfaces were cleaned by cycles of Ar ion bombardment (beam energy $1.3 \mathrm{keV}$ at $5 \times 10^{-6} \mathrm{mbar}$ of Ar, $10 \mathrm{~min}$ ) and subsequent annealing at $1200 \mathrm{~K}$ for $2 \mathrm{~min}$. If necessary, oxidation in $1 \times 10^{-6}$ mbar $\mathrm{O}_{2}$ was used to remove carbon contamination. The surface structure was examined by LEED. Before transferring to the SFG cell, the clean singlecrystal surface was exposed to $5 \times 10^{-7}$ mbar of $\mathrm{CO}$ gas at room temperature via a leak valve, in order to passivate the surface and to avoid unwanted adsorption of impurities on the surface during sample transfer.

SFG was performed using a Nd:YAG (neodymium-doped yttrium aluminum garnet; $\mathrm{Nd}: \mathrm{Y}_{3} \mathrm{Al}_{5} \mathrm{O}_{12}$ ) fundamental radiation of a PL2241 laser (EKSPLA, $1064 \mathrm{~nm}, 30 \mathrm{~mJ} / \mathrm{pulse}$ ), with a $20 \mathrm{ps}$ pulse width and $50 \mathrm{~Hz}$ repetition rate. Part of the fundamental laser is frequency converted to second harmonics $(532 \mathrm{~nm})$ realized in $\mathrm{K}^{*} \mathrm{DP}$ nonlinear crystals. One part of laser pulses at $532 \mathrm{~nm}$ is used as visible input for the surface SFG experiment. Another part of the $532 \mathrm{~nm}$ beam is used to pump an optical parametric (BBO crystal)/ difference frequency generation $\left(\mathrm{AgGaS}_{2}\right.$ crystal) system and generate tunable infrared light from $1000-4300 \mathrm{~cm}^{-1}$ $(2.3-10 \mu \mathrm{m})$ with a spectral width $<6 \mathrm{~cm}^{-1}$. The visible and infrared beams are spatially and temporally overlapped on the single-crystal surface in a co-propagating geometry at an incidence angle of $58.5^{\circ}$ and $55^{\circ}$ with respect to the surface normal, respectively. The energy is $\sim 40 \mu \mathrm{J} /$ pulse for the visible beam and energies between 90 and $130 \mu \mathrm{J} /$ pulse were used for the tunable infrared pulse with frequencies between 1800 and $2160 \mathrm{~cm}^{-1} . \mathrm{CaF}_{2}$ and quartz windows served as entrance and exit ports for the incident laser beams and output SFG radiation, respectively. The SFG signal is filtered with a monochromator and detected with a photo-multiplier tube (PMT). The polarization of the visible light and SFG signal were switched between $\mathrm{P}$ and $\mathrm{S}$ using a Glan-Taylor prism and a half-wave plate, while the infrared polarization was always kept as P (an S-polarized field would be canceled on a metal surface) [30] .

\section{Results and Discussion}

\subsection{SFG Spectra of CO on Pt(111)}

Polarization-dependent SFG spectra of CO/Pt(111) in the $\mathrm{C}-\mathrm{O}$ stretch region were acquired at mbar pressure and $300 \mathrm{~K}$. SSP and PPP spectra are presented in Fig. 1a. Because of the SFG selection rules [28], the SSP spectral intensity is much weaker than PPP. However, the spectra still had a good signal-to-noise ratio. The PPP spectrum of $\mathrm{CO}$ exhibits two peaks both attributed to linearly bonded $\mathrm{CO}$ molecules (with the carbon atom bound to a single $\mathrm{Pt}$ atom), but adsorbed at different sites of the single crystal. The sharp peak around $2092 \mathrm{~cm}^{-1}$ characterizes the on-top CO species adsorbed on terrace sites [31,32], whereas the smaller shoulder around $2073 \mathrm{~cm}^{-1}$ is assigned to species adsorbed on step-sites on the surface [24, 33]. Unlike the PPP spectrum, in the SSP spectrum only on-top CO molecules adsorbed on terrace sites were detected, while the step-sites CO was not clearly observed (likely due to lower surface number density). The asymmetric lineshape of the SFG spectra, which is particularly pronounced for the lower frequency band in the PPP spectrum, is due to the interference of resonant and non-resonant terms (Eq. 2); particularly when $\chi_{N R}$ is real and has the same sign as $\chi_{q}$ [34].

The correspondence between $\mathrm{C}-\mathrm{O}$ stretch frequencies and defect sites (steps or kinks) has been well documented for CO on Pt(111) using infrared spectroscopy [33, 34-38]. Frequencies for $\mathrm{CO}$ on defect sites are in general between 2058 and $2078 \mathrm{~cm}^{-1}$, lower than the frequencies of the terrace site vibrations. In a room-temperature SFG study of CO adsorbed on a disordered $\mathrm{Pt}(111)$ surface at a low coverage, a single vibrational frequency of $2072 \mathrm{~cm}^{-1}$ was reported by Daum and co-workers [24], but this frequency blue-shifted to $2093 \mathrm{~cm}^{-1}$ at higher coverage. For $\mathrm{CO}$ on a flat and wellordered $\mathrm{Pt}(111)$ surface, the frequency of the $\mathrm{C}-\mathrm{O}$ stretching vibration increases with exposure from 2083 to $2093 \mathrm{~cm}^{-1}$ at $300 \mathrm{~K}$ [24]. In addition, $\mathrm{CO}$ molecules prefer to adsorb on the step sites first, and then on the terrace-site of a defectrich $\mathrm{Pt}(111)$ surface [33]. Similarly, studies of other surfaces with a high step density, such as $\operatorname{Pt}(533)$, also indicated that the adsorption of CO initially occurs in a linearly bonded configuration at step sites [37]. Thus, the appearance of a peak at $2073 \mathrm{~cm}^{-1}$ in Fig. 1a is attributed to the existence of steps on $\mathrm{Pt}(111)$.

Adsorbates on metal surfaces are usually considered not to produce (S or P) SFG signals for the SP (S-vis, P-IR) 
(a)

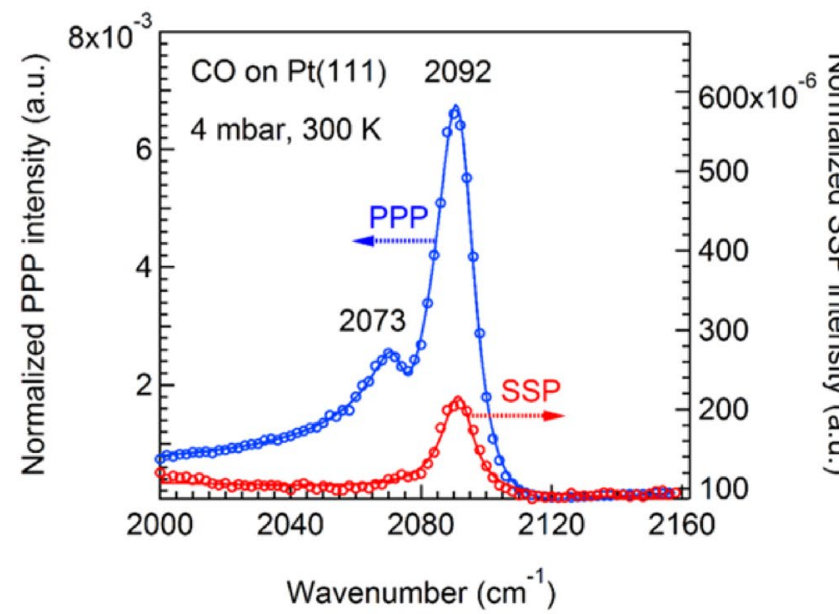

(b)

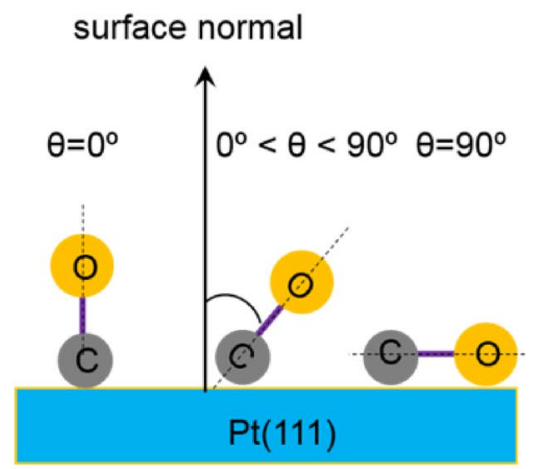

Fig. 1 a Polarization-dependent SFG spectra of CO adsorption on $\mathrm{Pt}(111)$ at 4 mbar and $300 \mathrm{~K}$. Symbols refer to experimental data. Blue circles: PPP polarization; Red circles: SSP polarization; Solid

combination if the adsorbates have vibrational modes oscillating only along the surface normal [39]. The perpendicularly adsorbed CO on Pt(111) should thus not yield an SSP signal. However, in agreement with previous studies [22, 23] (in which some of us have demonstrated that this simplification is not valid), our experiment showed that both SSP and PPP spectra of on-top CO at terrace sites of the Pt(111) single crystal had a good signal-to-noise ratio. Fitting the peaks with Lorentzian lineshapes using Eq. 2 provided an accurate $I_{\mathrm{ppp}} / I_{\mathrm{ssp}}$ value of 27 for the on-top CO stretch mode. Next, we used the ratio of $I_{\mathrm{ppp}} / I_{\mathrm{spp}}$ to analyze the orientation (tilt) angle (definition is illustrated in Fig. 1b) or molecular hyperpolarizability ratio $\left(R=\beta_{a a c} / \beta_{c c c}=\beta_{b b c} / \beta_{c c c}\right)$ of on-top $\mathrm{CO}$ at the gas $/ \operatorname{Pt}(111)$ interface.

\subsubsection{Simulated $I_{\mathrm{ppp}} / I_{\mathrm{ssp}}$ Versus Tilt Angle $(\theta)$ and Hyperpolarizability Ratio ( $R$-Value)}

According to Eq. 4, the value alone is not sufficient to calculate the orientation angle of a certain surface molecule or functional group, because the hyperpolarizability ratio ( $R$-value) must be known as well. Because SSP spectra of $\mathrm{CO}$ on single-crystal surfaces were rarely obtained, and the orientation angle of on-top $\mathrm{CO}$ was generally assumed to be $0^{\circ}[22,23]$, the orientation analysis has never been discussed in depth before. Almost two decades ago, only Badelli et al. [23] deduced an $R$-value of 0.6 and 1.5 for on-top CO and bridge-CO species on $\mathrm{Pt}(111)$ electrodes, respectively, based on the ratio of $I_{\mathrm{ssp}} / I_{\mathrm{ppp}}$ for CO molecules oriented parallel to the surface normal.

Since both the tilt angle $\theta$ and $R$-value are unknown parameters, we theoretically simulated the ratio of $I_{\mathrm{ppp}} / I_{\mathrm{ssp}}$ lines represent the global fitting curves with Lorentzian lineshapes (Eq. 2). b Schematic diagram of tilt angles of CO on a Pt single crystal surface

as a function of tilt angle with different $R$-values (Fig. 2a) and, vice versa, as a function of $R$-value assuming a $0^{\circ}$ tilt angle (Fig. 2b). Refractive indices used in the simulations are summarized in Table 1. Incidence angles of visible $(532 \mathrm{~nm})$ and infrared $\left(2092 \mathrm{~cm}^{-1}\right)$ beams in the simulation were $58.5^{\circ}$ and $55^{\circ}$, respectively.

For a $\mathrm{CO}$ molecule with a $C_{\mathrm{cov}}$ symmetry, the $R$-value equals to its bond polarizability derivative ratio $r$. Generally, $0 \leq r<1$ applies for a single chemical bond [28]. The $I_{\mathrm{ppp}} / I_{\mathrm{ssp}}$ value of 27 , obtained from our SFG results, corresponds to $\mathrm{R} \approx 0.5$ (see Fig. $2 \mathrm{a}$ ), assuming a $0^{\circ}$ tilt angle, which is close to the $0.6 R$-value reported earlier for on-top $\mathrm{CO}$ [23]. The difference of the calculated $R$-value may be due to different values of refractive indices of bulk and/or interfacial Pt (Ref. [23] did not specify them). However, the on-top $\mathrm{CO}$ molecules may not stand exactly upright on the Pt atoms. If we keep $I_{\mathrm{ppp}} / I_{\mathrm{ssp}}=27$, the tilt angle of on-top $\mathrm{CO}$ is $40^{\circ}, 30^{\circ}, 22^{\circ}$ and $0^{\circ}$ in the case of adopting $R$-values of $0.1,0.3,0.4$ and 0.5 , respectively. When $R=0.6$, there is no intersection at any tilt angle, according to our experimental result. Based on the discussion above, due to the uncertainty of $R$-values, it is difficult to determine the precise orientation of $\mathrm{CO}$ molecules on $\mathrm{Pt}$ surfaces without complementary information from other methods. For example, the Raman depolarization ratio $(\rho)$ and bond polarizability derivative model have already been used to obtain a quantitative (but not fully accurate) description of the hyperpolarizability tensor ratios $(R)$ in SFG analysis for stretching vibrational modes of the $\mathrm{CH}_{3}$, $\mathrm{CH}_{2}$ and $\mathrm{CH}$ groups $[21,41,42]$. The $\rho$-value can be accurately measured with polarized Raman techniques [28, 43, $44]$ and the $R$-value can be deduced from the $\rho$-value, for 

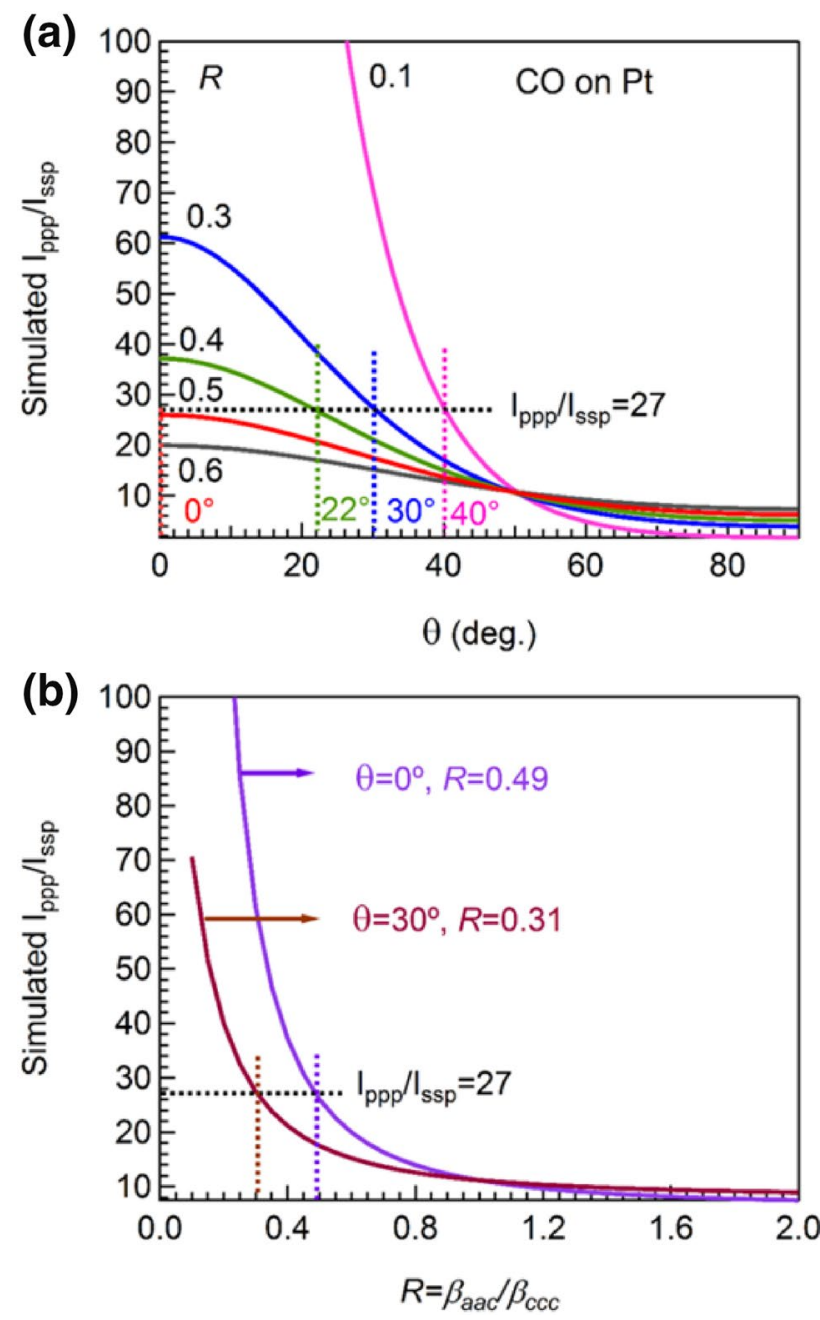

Fig. 2 Simulated $I_{\mathrm{ppp}} / I_{\mathrm{ssp}}$ ratio as a function of a tilt angle $(\theta)$ for the $\mathrm{CO}$ molecule with a $\mathrm{C}_{\infty \mathrm{v}}$ symmetry and $\mathbf{b}$ molecular hyperpolarizability ratio $(R)$. The following parameters were used in the simulation: $\omega_{I R}=2090 \mathrm{~cm}^{-1}, \alpha_{I R}=55^{\circ} ; \omega_{V i s}=532 \mathrm{~nm}, \alpha_{V i s}=58.5^{\circ} ; N_{s}=1, \beta_{\text {ccc }}=1$. All refractive indices used for simulation are shown in Table 1

$C_{\infty v}$ symmetry, the relationship between $R$ or $r$-value and $\rho$-value is $\rho=3 /\left(4+5[(1+2 r) /(1-r)]^{2}\right)$ [28]. Thus, if one can get the Raman depolarization ratio of $\mathrm{CO}$ molecules directly via experiments or theoretical calculations, the molecular hyperpolarizability or bond polarizability tensor ratio can be obtained, and the orientation analysis of adsorbed $\mathrm{CO}$ molecules becomes feasible. However, the $\rho$-value of CO molecules has not yet been reported.
Similarly, if the orientation angle is known, the precise $R$-value can also be determined. As the ratio of $I_{\mathrm{ppp}} / I_{\mathrm{ssp}}$ decreases, the $R$-value increases (Fig. $2 \mathrm{~b}$ ). More specifically, when $I_{\mathrm{ppp}} / I_{\mathrm{ssp}}>40$, the $R$-value is hardly sensitive to changes of $I_{\mathrm{ppp}} / I_{\mathrm{ssp}}$; whereas a small drop of the intensity ratio between 0 and 40 corresponds to a significant increase of the $R$-value. However, when the value of $I_{\mathrm{ppp}} / I_{\mathrm{spp}}$ is smaller than 10 , no reasonable $R$-value can be obtained anymore. Taking tilt angles of $0^{\circ}$ and $30^{\circ}$ as example, the exact $R$-value is 0.49 and 0.31 (Fig. 2b), respectively. Obviously, only 0.18 difference of $R$-value may lead to $30^{\circ}$ tilt angle deviations, indicating that the determination of the orientation of surface $\mathrm{CO}$ molecules is rather difficult.

\subsubsection{Pressure-Dependent SFG Spectra of CO Bond Stretching}

Taking advantage of the high pressure capability of SFG, pressure-dependent spectra of $\mathrm{CO}$ adsorption on $\mathrm{Pt}(111)$ were also measured (Fig. 3). The SSP and PPP spectral intensities simultaneously changed with the $\mathrm{CO}$ pressure, yielding almost the same $I_{\mathrm{ppp}} / I_{\mathrm{ssp}}$ ratio $(20 \pm 2)$ at different pressures. Apparently, the orientation of $\mathrm{CO}$ did not change at $300 \mathrm{~K}$ in the studied pressure range. Similarly, it was previously reported that there was no significant variation of the tilt angle upon increasing coverage of CO molecules adsorbed on (100) surfaces of bodycentered cubic transition metals ( $\mathrm{Fe}, \mathrm{Mo}, \mathrm{Cr}$, and W) [45]. The peak position of $\mathrm{CO}$ at high pressure is shifted about $16 \mathrm{~cm}^{-1}$, as compared to those of Fig. 1. This can be explained by the $\mathrm{CO}$-induced roughening of the surface, an effect that has also been reported by the Somorjai group [46]. CO-induced roughening was also observed for supported Pt nanoparticles [47].

There were only small changes in intensity and frequency upon increasing the pressure, since at $300 \mathrm{~K}$ saturation is almost reached at $10^{-4} \mathrm{mbar}$. This agrees with previous results of $\mathrm{CO}$ on smooth $\mathrm{Pt}(111)$ [31]. The observed increase of spectral intensity in PPP and SSP spectra can be attributed to the increasing surface coverage at constant orientation angle.

The absence of a vibrational band of bridge-bound $\mathrm{CO}$ on $\mathrm{Pt}(111)$ at $300 \mathrm{~K}$ can be explained by the strong broadening of the linewidth $2 \Gamma$ at high temperature [24]. Schweizer et al. [48], for example, had reported that $\mathrm{CO}$ on bridge sites had a linewidth of $37 \mathrm{~cm}^{-1}$ at $300 \mathrm{~K}$. The linewidth broadening has been attributed to an order-disorder transition [49] with a partial loss of local order of the CO adlayer around $300 \mathrm{~K}$ [48].
Table 1 Bulk and interface refractive indices of air, $\mathrm{Pt}$, and $\mathrm{Pd}$ at different wavelengths

\begin{tabular}{lllllll}
\hline & $\lambda(\mu \mathrm{m})$ & $\mathrm{n}_{1}($ air $)$ & $\mathrm{n}_{2}(\mathrm{Pt})[40]$ & $\mathrm{n}^{\prime}(\mathrm{Pt})$ & $\mathrm{n}_{2}(\mathrm{Pd})[40]$ & $\mathrm{n}^{\prime}(\mathrm{Pd})$ \\
\hline SFG & 0.479 & 1 & $1.91+3.3 \mathrm{i}$ & $1.14+1.4 \mathrm{i}$ & $1.47+3.4 \mathrm{i}$ & $0.89+1.4 \mathrm{i}$ \\
Vis & 0.532 & 1 & $2.04+3.6 \mathrm{i}$ & $1.18+1.6 \mathrm{i}$ & $1.60+3.7 \mathrm{i}$ & $0.94+1.6 \mathrm{i}$ \\
IR & 4.785 & 1 & $3.89+18.9 \mathrm{i}$ & $1.96+9.4 \mathrm{i}$ & $3.3+20.2 \mathrm{i}$ & $1.66+10.0 \mathrm{i}$ \\
\hline
\end{tabular}



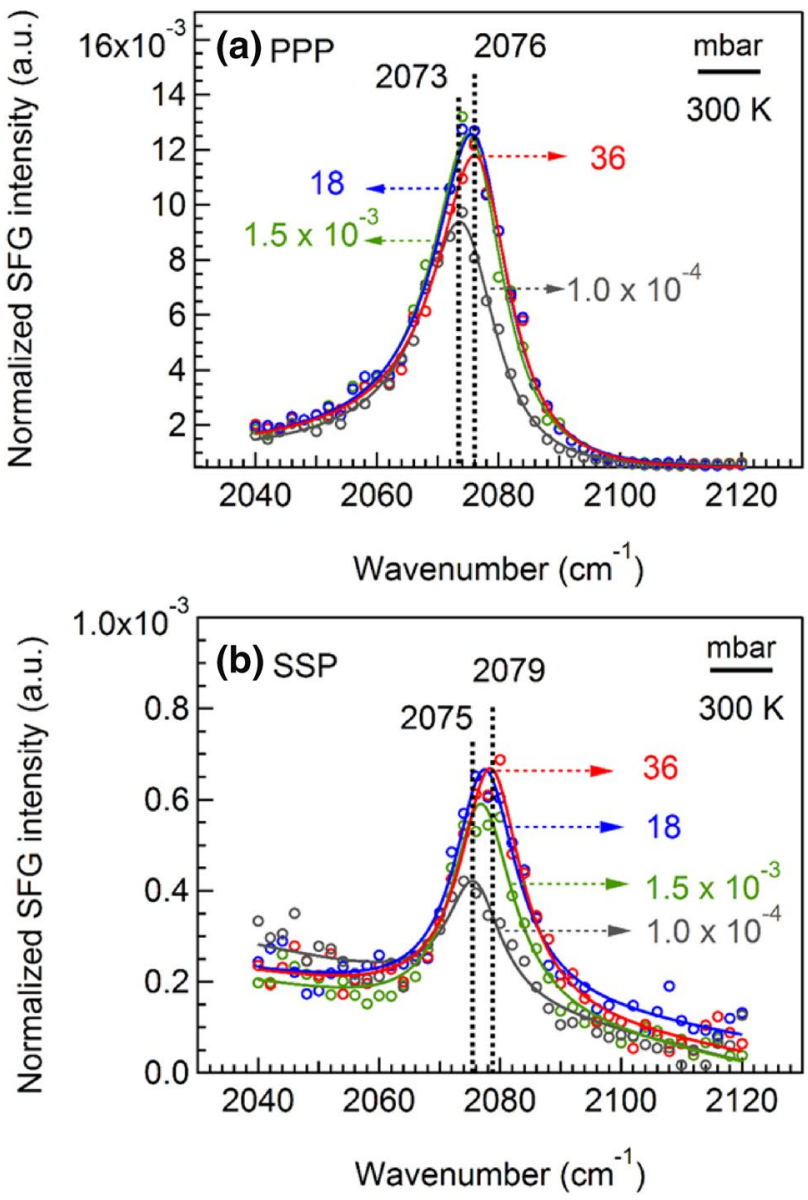

Fig. 3 Pressure-dependent a PPP and b SSP spectra of CO on Pt(111) at $300 \mathrm{~K}$

\subsubsection{The Effect of Incidence Angles of Visible and IR Laser Beams}

SSP spectra of CO on $\mathrm{Pt}(111)$ have been rarely reported in previous SFG studies. However, as reported by Baldelli et al. [23] and herein, both SSP and PPP spectra with good signal-to-noise ratio can be acquired. In Ref. [23], a $I_{\mathrm{ppp}} / I_{\mathrm{ssp}}$ value of 1.25 (comparable spectral intensity of SSP and PPP) was obtained using incidence angles of $33^{\circ}$ and $45^{\circ}$ for visible and IR laser beams, respectively. As mentioned above, our results showed $I_{\mathrm{ppp}} / I_{\mathrm{ssp}}$ was 27 (spectral intensity of SSP is much smaller than PPP) for the experimental configuration of $55^{\circ}$ and $58.5^{\circ}$ for visible and IR laser beams, respectively. Obviously, the relative magnitude of the spectral intensity has a strong dependence on the incidence angles of the laser beams. In order to examine the effect of incidence angles on SFG spectra, we simulated $I_{\mathrm{ppp}}, I_{\mathrm{ssp}}$ (Fig. 4a) and $I_{\mathrm{ppp}} / I_{\mathrm{ssp}}$ of CO molecules on Pt (assuming $\theta=0^{\circ}$ and $R=0.49$ ) for different incidence angles $\left(\alpha_{V i s}\right.$ and $\left.\alpha_{I R}\right)$. For better display, the curves of $I_{\mathrm{ppp}} / I_{\mathrm{ssp}}$ versus $\alpha_{I R}$ (Fig. $4 \mathrm{~b}$ ) and $\alpha_{V i s}$ are shown in separate diagrams, and $I_{\mathrm{ppp}} / I_{\mathrm{ssp}}$ versus $\alpha_{V i s}$ is even divided into two regions: one in the range of $\alpha_{V i s}=0^{\circ}-75^{\circ}$ (Fig. 4c), and another in the range of $\alpha_{V i s}=75^{\circ}-90^{\circ}$ (Fig. 4d). The parameters of refractive indices, used for the simulations, are shown in Table 1.

We first consider the spectral intensity changes. In Fig. $4 \mathrm{a}$, for $\alpha_{V i s}=55^{\circ}, I_{\mathrm{ppp}}$ and $I_{\text {ssp }}$ showed a strong but similar dependence on $\alpha_{I R}$, and reach the maximum for $\alpha_{I R}=72^{\circ}$. The ratio of $I_{\mathrm{ppp}} / I_{\text {ssp }}$ is less sensitive to $\alpha_{I R}$ (Fig. 4b), as a result of the similar intensity response to $\alpha_{I R}$, which is consistent with a previous report [50]. However, for $\alpha_{I R}=55^{\circ}, I_{\mathrm{ppp}}$ and $I_{\mathrm{ssp}}$ depended on $\alpha_{V i s}$ differently, $I_{\mathrm{ppp}}$ changed non-monotonically (increased first and then decreased), whereas $I_{\text {ssp }}$ decreased monotonically, resulting in a gradually increasing $I_{\mathrm{ppp}} / I_{\mathrm{ssp}}$ ratio (Fig. 4c, d).

In more detail, as evident from Fig. $4 \mathrm{c}$, d, the value of $I_{\mathrm{ppp}} / I_{\mathrm{ssp}}$ has a very strong dependence on $\alpha_{V i s}$ at fixed $\alpha_{I R}$. A continuous increase of $I_{\mathrm{ppp}} / I_{\mathrm{ssp}}$ with increasing $\alpha_{V i s}$ indicates that the SSP signal will be rather difficult to obtain (as compared to PPP polarization), when a larger $\alpha_{V i s}$ is used. For example, when $\alpha_{V i s}$ is in the range of $0^{\circ}-60^{\circ}$, $I_{\mathrm{ppp}} / I_{\mathrm{ssp}}$ is smaller than 30 (Fig. $4 \mathrm{c}$ ), and $I_{\mathrm{ppp}}$ reaches the maximum value at $\alpha_{V i s}=60^{\circ}$; however, when $\alpha_{V i s}$ is larger than $70^{\circ}$, there are more than two orders of magnitude difference between $I_{\mathrm{ppp}}$ and $I_{\mathrm{ssp}}$ (Fig. 4d), e.g. when $\alpha_{V i s}=80^{\circ}$, $I_{\mathrm{ppp}}$ is (unexpectedly) 309 times larger than $I_{\text {ssp }}$. Increasing $\alpha_{V i s}$ further, the ratio of $I_{\mathrm{ppp}} / I_{\mathrm{ssp}}$ could reach more than 800 (Fig. 4d), and $I_{\text {ppp }}$ decreases gradually (Fig. 4a, green solid line). This shows that especially $\alpha_{V i s}$ plays a crucial role in polarization-dependent SFG studies. A previous study of the effects of refractive index and $\alpha_{V i s}$ on the sum frequency generation intensity at air/liquid and solid/liquid interfaces also demonstrated the significance of $\alpha_{V i s}$ [51].

In analogy to using the ratio of $I_{\mathrm{ppp}} / I_{\mathrm{ssp}}$ at a specific experimental configuration, the $R$-value at a selected tilt angle can also be deduced by measuring the ratio of $I_{\mathrm{ppp}}$ collected from different experimental configurations. The two methods could then be used to mutually verify each other. Accordingly, for $R=0.49$ (deduced from $I_{\mathrm{ppp}} / I_{\mathrm{ssp}}=27$ ), the simulated ratio of $I_{\mathrm{ppp}}$ of Configuration $1\left(\alpha_{I R}=55^{\circ}, \alpha_{V i s}=58.5^{\circ}\right)$ and $I_{\mathrm{ppp}}$ of Configuration 2 $\left(\alpha_{I R}=45^{\circ}, \alpha_{V i s}=37^{\circ}\right)$ would be 2.11, as shown in Fig. 5 . Unfortunately, due to limitations of our UHV-compatible SFG setup, the incidence angles of IR and visible beams can be only adjusted within a few degrees. We are thus currently unable to experimentally determine the ratio of $\mathrm{I}_{\mathrm{ppp}}$ at different experimental configurations and to confirm the $R$-value that was deduced from $\mathrm{I}_{\mathrm{ppp}} / \mathrm{I}_{\mathrm{ssp}}$. However, the suggested method may be applied in the future using SFG setups that can widely vary the experimental configuration. 

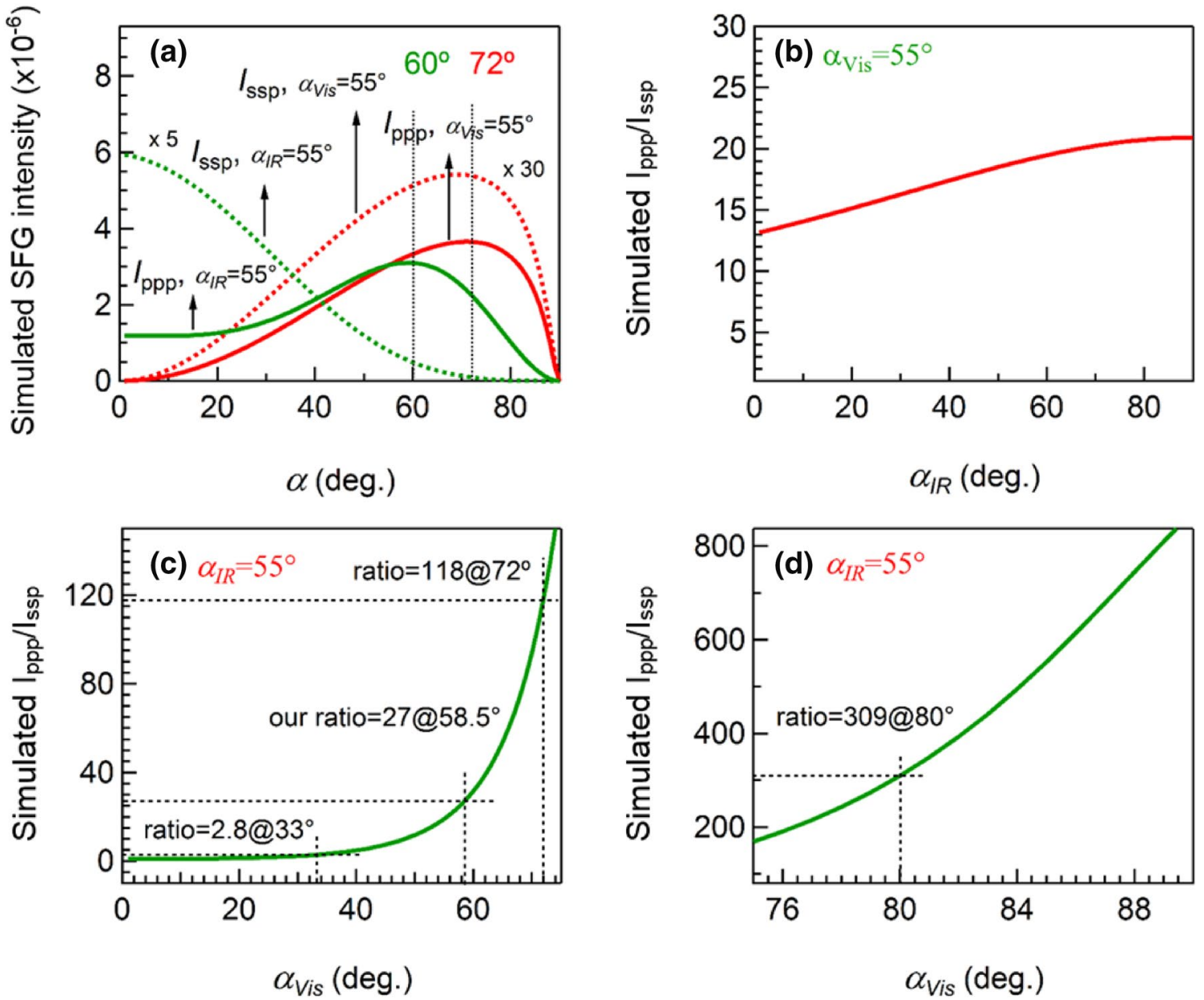

Fig. 4 Simulations: a $I_{\mathrm{ppp}}$ and $I_{\mathrm{ssp}}$ versus incidence angles: fixed $\alpha_{V i s}=55^{\circ}, I_{\mathrm{ppp}}$ (red solid line) and $I_{\mathrm{ssp}}$ (red dashed line) versus $\alpha_{I R}$ and fixed $\alpha_{I R}=55^{\circ}, I_{\mathrm{ppp}}$ (green solid line) and $I_{\text {ssp }}$ (green dashed line) versus $\alpha_{V i s} . \mathbf{b} I_{\mathrm{ppp}} / I_{\mathrm{ssp}}$ versus $\alpha_{I R}$ (fixed $\alpha_{V i s}=55^{\circ}$, red solid line). c $I_{\mathrm{ppp}} / I_{\mathrm{ssp}}$ versus $\alpha_{V i s}$ (fixed $\alpha_{I R}=55^{\circ}$, green solid line), $\alpha_{V i s}$

$=0-75^{\circ}$. d $I_{\mathrm{ppp}} / I_{\mathrm{ssp}}$ versus $\alpha_{V i s}\left(\right.$ fixed $\alpha_{I R}=55^{\circ}$, green solid line $), \alpha_{V i s}$ $=75-90^{\circ}$. The following parameters were used in the simulation: $\omega_{I R}=2090 \mathrm{~cm}^{-1}, \omega_{V i s}=532 \mathrm{~nm} ; N_{s}=1, \beta_{c c c}=1, \theta=0^{\circ}$ and $R=0.49$. All refractive indices used for simulation are shown in Table 1

\subsection{SFG Spectra of CO on Pd(111)}

$\mathrm{Pd}$ is used in many catalytic processes such as hydrogenation, oxidation/combustion, etc. [52,53], and CO adsorption has also been used to characterize mono- and bimetallic surfaces $[54,55]$. The pressure- and temperature-dependent $\mathrm{CO}$ adsorption on $\mathrm{Pd}(111)$ has thus been extensively studied, mostly using PPP polarization [56-59]. However, PPP and SSP spectra of $\mathrm{CO} / \mathrm{Pd}(111)$ with good signal-to-noise ratio $\left(I_{\mathrm{ppp}} / I_{\mathrm{ssp}}=10\right)$ were also obtained at $90 \mathrm{~K}$, for an experimental configuration of $\alpha_{V i s}=54^{\circ}$ and $\alpha_{I R}=56^{\circ}$ [22] (similar to the current experimental conditions: $\alpha_{V i s}=58.5^{\circ}, \alpha_{I R}$ $\left.=55^{\circ}\right)$. At the low temperature $(90 \mathrm{~K})$, the $0.75 \mathrm{ML} 2 \times 2$ saturation structure of $\mathrm{CO}$ was (predominantly) present and the signal of on-top CO $\left(2108 \mathrm{~cm}^{-1}\right)$, both in SSP and PPP spectra was much stronger than the signal of hollow $\left(1890 \mathrm{~cm}^{-1}\right)$ and bridge $\left(1925 \mathrm{~cm}^{-1}\right)$ bonded CO. At higher

temperature $(200 \mathrm{~K})$, i.e. lower coverage, the signal of bridge $\left(1951 \mathrm{~cm}^{-1}\right)$ and on-top $\left(2081 \mathrm{~cm}^{-1}\right)$ CO species became comparable in PPP, whereas no adsorbate species could be detected in the SSP spectra.

Herein, we have acquired PPP and SSP spectra for CO adsorption on $\mathrm{Pd}(111)$ at $300 \mathrm{~K}$, complementing the measurements in Ref. [22], and have also used higher $\mathrm{CO}$ gas pressure (Fig. 6). Apparently, the hollow peak at $\sim 1899 \mathrm{~cm}^{-1}$ (present as shoulder) and the bridge peak $\left(1923 \mathrm{~cm}^{-1}\right)$ rather than the on-top peak $\left(2071 \mathrm{~cm}^{-1}\right)$ dominated the PPP spectra [56-59] (due to coverage lower than that of the low-temperature PPP spectra in Ref. [22]). From $1 \times 10^{-6}$ mbar to 250 mbar an approximate $12 \mathrm{~cm}^{-1}$ blue shift was observed for bridge-bonded $\mathrm{CO}$ due to increased coverage. For on-top $\mathrm{CO}$ a second peak appeared at $2053 \mathrm{~cm}^{-1}$ in 6 mbar. Apparently, at room temperature the $\mathrm{CO}$ overlayer is not perfectly ordered but rather a superposition of several $\mathrm{CO}$ structures in 


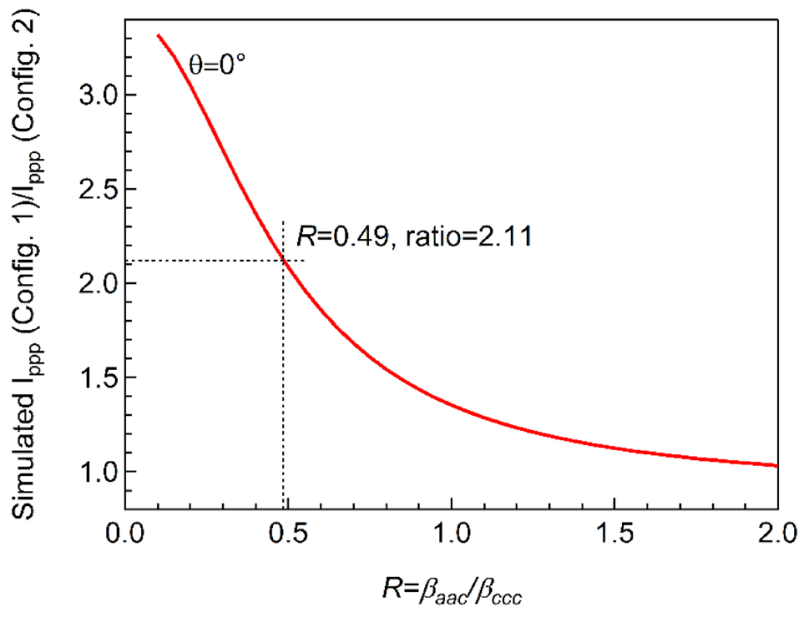

Fig. 5 Simulated ratio of $I_{\text {ppp }}$ of Configuration 1 $\left(\alpha_{I R}=55^{\circ}, \alpha_{V i s}=58.5^{\circ}\right)$ and $I_{\mathrm{ppp}}$ of Configuration 2 $\left(\alpha_{I R}=45^{\circ}, \alpha_{V i s}=37^{\circ}\right)$ as a function of molecular hyperpolarizability ratio (R) assuming $\theta=0^{\circ}$. The following parameters were used in the simulation: $\omega_{I R}=2090 \mathrm{~cm}^{-1}, \omega_{V i s}=532 \mathrm{~nm} ; \mathrm{N}_{\mathrm{s}}=1, \beta_{\mathrm{ccc}}=1$. All refractive indices used for simulation are given in Table 1

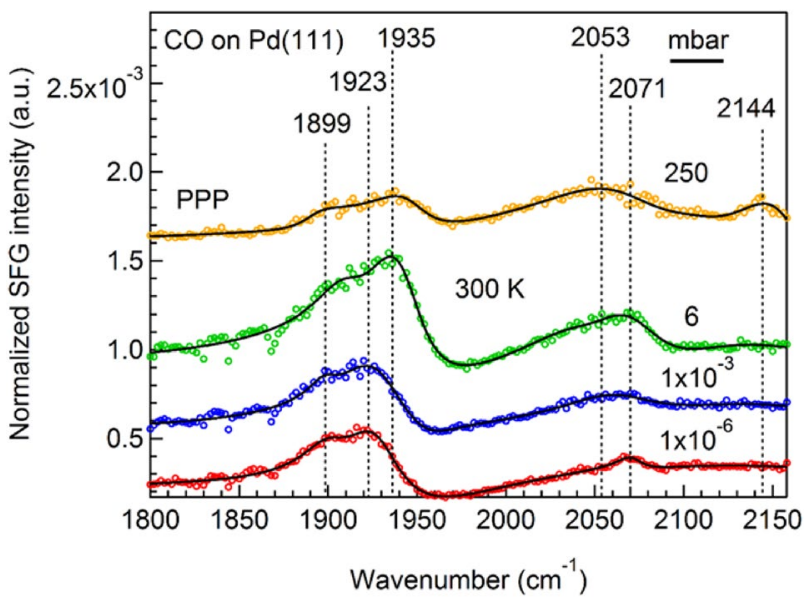

Fig. 6 PPP spectra of CO adsorption on $\mathrm{Pd}(111)$ at $300 \mathrm{~K}$

the coverage range $0.5-0.6 \mathrm{ML}$. For more detailed descriptions see Refs. [59, 60].

At 250 mbar, on-top CO not only existed in two species but there was also another higher frequency "feature" at $2144 \mathrm{~cm}^{-1}$ in PPP. Previous infrared reflection-adsorption spectra (IRAS) of 50 mbar CO on Pd(111) at $300 \mathrm{~K}$ also displayed a $\mathrm{C}-\mathrm{O}$ vibrational feature, which is due to gas phase $\mathrm{CO}$ absorption $\left(2143 \mathrm{~cm}^{-1}\right)$ [61].

As shown in Fig. 6, the SFG spectral intensity gradually increased from low pressure to 6 mbar due to increasing surface $\mathrm{CO}$ coverage, but the spectral intensity strongly decreased at 250 mbar. As illustrated by Eq. 1, the SFG intensity depends on the effective surface IR and visible intensities. Although no SFG signal is generated by gas phase CO, the SFG process is indirectly influenced by the loss of IR-light via absorption by high-pressure $\mathrm{CO}$ gas. Thus, the (on-top) SFG signal may drop because the IR energy arriving at the sample is reduced in higher pressure CO. All SFG spectra were normalized by the IR and Vis energies but the energy-detector was located outside the input window, thus this normalization does not account for the $\mathrm{CO}$ gas phase absorption, which explains the unusual peak at $2144 \mathrm{~cm}^{-1}$ in PPP. It can be attributed to the $5.4 \mathrm{~cm}$ distance between the input window and the sample surface. In Refs. [56, 57, 59], using recessed IR windows, the distance was only $1.5 \mathrm{~cm}$, so no IR absorption was observed even in 1000 mbar CO gas. For the current setup, normalization could be achieved by using GaAs references, by measuring the reflected IR light after the exit window, or by putting a $5.4 \mathrm{~cm}$ gas cell in front of the IR detector [2].

Unfortunately, in SSP polarization the peak intensities were too small for $\mathrm{CO}$ on Pd to be detected. In the following, we will explain the specific reason by comparing experimental and simulation results for $\mathrm{CO}$ on $\mathrm{Pt}$ and $\mathrm{Pd}$.

\subsection{Comparison of $\mathrm{CO}$ on $\mathrm{Pt}(111)$ and $\mathrm{Pd}(111)$}

In order to better illustrate the observed intensity differences, we have plotted the PPP spectra of CO on Pt(111) and $\operatorname{Pd}(111)$ together with some spectra from Refs [56, 59]. (Fig. 7a). Since on-top CO species were observed on both surfaces, we focus on disscussing SFG intensity changes of on-top CO. The PPP spectral intensity of CO on Pt(111) is obviously much larger than that on $\operatorname{Pd}(111)$. However, the on-top CO intensity on $\mathrm{Pd}(111)$ is strongly coverage dependent and we have thus included spectra at 10 and 400 mbar, when the higher $\mathrm{CO}$ coverage leads to intense $\mathrm{CO}$ peaks.

To better understand the distinctive intensity differences of the SFG experiments, we simulated $I_{\text {ppp }}$ (Fig. 7b), $I_{\text {ssp }}$ (Fig. 7c) and $I_{\mathrm{ppp}} / I_{\mathrm{ssp}}$ (Fig. 7d) versus $R$ for on-top CO on $\mathrm{Pt}$ and Pd surfaces. For simulations, we assumed the same values of tilt angle $\theta=0^{\circ}$, effective surface number density $N_{s}=1$ and molecular hyperpolarizability $\beta_{c c c}=1$ for Pt and Pd single crystals. Refractive indices used in the simulation can be found in Table 1. Interestingly, according to the theoretical simulation results, $I_{\mathrm{ppp}}(\mathrm{Pd})$ is about three times larger than $I_{\mathrm{ppp}}(\mathrm{Pt})$, and $I_{\mathrm{ssp}}(\mathrm{Pd})$ is about two times larger than $I_{\mathrm{ssp}}$ (Pt) in the whole range of $R=0.1-2$. The simulated results are thus different from the experimental ones, for which $\mathrm{Pt}$ typically exhibits stronger SFG signal. Nevertheless, both experiment and theory deviate not too far in the order of magnitude of the SFG signal.

According to SFG theory, the factors influencing the SFG intensity of molecules adsorbing on different surfaces under the same experimental conditions (mainly referring to incidence angles of IR and visible beams), include the 

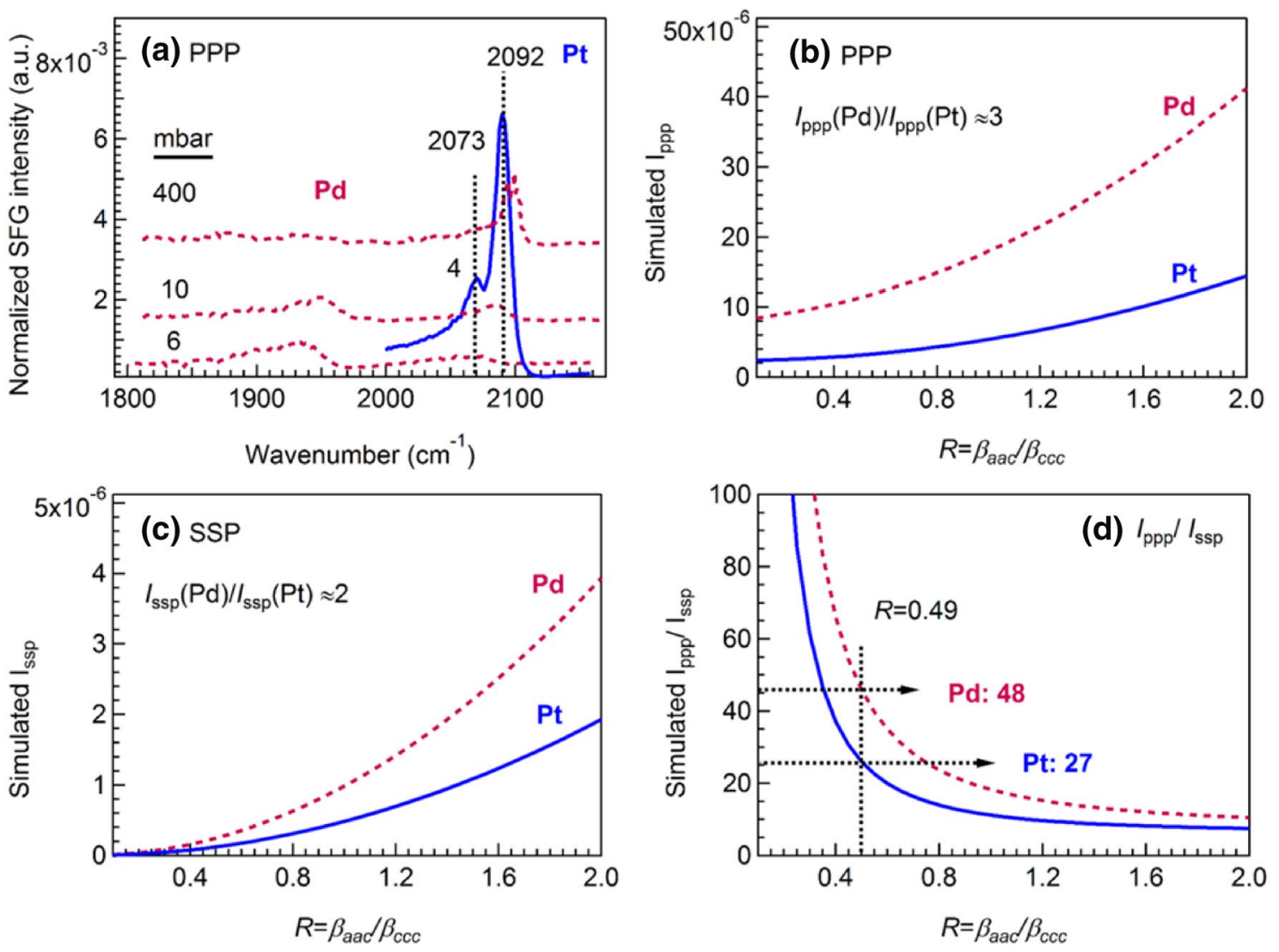

Fig. 7 Comparison of spectral intensity of on-top $\mathrm{CO}$ on $\mathrm{Pd}(111)$ and $\mathrm{Pt}(111)$. a Experimental PPP spectra at $300 \mathrm{~K}, I_{\mathrm{ppp}}(\mathrm{Pd})<I_{\mathrm{ppp}}$ (Pt). Dark red: CO on Pd(111): 6 mbar (our data), 10 and 400 mbar (adapted from Refs. [56, 59]); blue: $\mathrm{CO}$ on $\mathrm{Pt}(111)$ : 4 mbar. b Simu-

effective surface number density $\left(N_{\mathrm{s}}\right)$, molecular orientation angles $(\theta)$, polarizations (e.g. SSP, PPP, etc.), Fresnel factors $\left[L_{\mathrm{ii}}\right.$, related to laser incidence angles and refractive indices of bulk phases $(n)$ and interfacial layer $\left.\left(n^{\prime}\right)\right]$, molecular hyperpolarizability tensor $\left(\beta_{c c c}\right)$ and tensor ratio $\left(R=\beta_{a a d} / \beta_{c c c}=\beta_{b b c} / \beta_{c c c}\right)$. All possible changes of the influence factors were considered for $\mathrm{CO}$ adsorption on $\mathrm{Pt}$ and $\mathrm{Pd}$ surfaces, except assuming identical $N_{\mathrm{s}}$. Thus, the conflicting results of experiments $\left[I_{\mathrm{ppp}}(\mathrm{Pd})<I_{\mathrm{ppp}}(\mathrm{Pt})\right]$ and simulations $\left[I_{\mathrm{ppp}}(\mathrm{Pd})>I_{\mathrm{ppp}}(\mathrm{Pt})\right]$, plus the fact that the intensity of an SFG peak is propotional to the square of $N_{\mathrm{s}}$, implies that the $N_{\mathrm{s}}$ of $\mathrm{CO}$ on the Pd surface should be much smaller than that on the Pt surface.

Assuming that the molecular hyperpolarizability ratio ( $R$-value) of CO molecules is identical on different single crystal surfaces (based on the result of $R=0.49$ and assumed $\theta=0^{\circ}$ ) for $\mathrm{CO}$ on $\mathrm{Pt}$, the theoretical value of $I_{\mathrm{ppp}} / I_{\mathrm{spp}}$ would be 48 for the Pd surface. This is much larger than the value of 27 for CO on Pt. Taking into account the small PPP signal on Pd (Fig. 7a) and the simulated large value of $I_{\mathrm{ppp}} / I_{\mathrm{ssp}}$, this indicates that SSP spectra of on-top CO on Pd(111) are

lated $I_{\mathrm{ppp}}$ versus $R$. c Simulated $I_{\mathrm{ssp}}$ versus $R$. d $I_{\mathrm{ppp}} / I_{\mathrm{ssp}}$ versus $R$. The following parameters were used in the simulation: $\omega_{I R}=2090 \mathrm{~cm}^{-1}$, $\alpha_{I R}=55^{\circ} ; \omega_{V i s}=532 \mathrm{~nm}, \alpha_{V i s}=58.5^{\circ} ; N_{s}=1, \beta_{\mathrm{ccc}}=1$, and $\theta=0^{\circ}$. All refractive indices used for simulation are shown in Table 1

difficult to measure at room temperature. Consequently, no reasonable SSP spectra could be acquired.

\section{Conclusions}

Polarization-dependent sum frequency generation (SFG) vibrational spectroscopy is a useful method, providing interface-specific spectra of adsorbed molecules, even at elevated gas pressure. For $\mathrm{CO}$ overlayers on $\mathrm{Pt}(111)$ and $\mathrm{Pd}(111)$ single crystal surfaces at room temperature, different polarization combinations (SSP and PPP) of the visible and SFG light were utilized to determine the molecular orientation (tilt angle) of $\mathrm{CO}$. However, since the hyperpolarizability ratio $\left(R=\beta_{a a c} / \beta_{c c c}=\beta_{b b c} / \beta_{c c c}\right)$ is not exactly known, we have rather determined this value $(R=0.49)$ by assuming perpendicular adsorption of $\mathrm{CO}$ (tilt angle of $0^{\circ}$ ). Pressuredependent SFG spectra on $\operatorname{Pt}(111)\left(10^{-4}\right.$ to $\left.36 \mathrm{mbar}\right)$ did not indicate any orientation change of adsorbed $\mathrm{CO}$. Modeling the expected SFG ratio indicated a strong dependence on 
the experimental configuration, especially on the incidence angle of the visible beam.

Room temperature CO adsorption on $\mathrm{Pd}(111)$ was examined in the pressure range of $10^{-6}$ to 250 mbar but only PPP and no SSP spectra could be acquired. Modeling the absolute PPP and SSP spectral intensities on Pt and Pd, as well as the expected ratios, explained the absence of SSP signals on Pd. Care must apparently be taken even for the orientation analysis of seemingly simple adsorbate-substrate systems, with the interface model not only including refractive indices, but also the experimental configuration (incidence angles) and surface coverages.

Acknowledgements Open access funding provided by TU Wien (TUW). G. R. acknowledges TU Wien for the Innovative Project "SFG Spectroscopy". This work was supported by the Austrian Science Fund (FWF) under projects I 1041-N28 (ComCat), I 942-N17 (DryRef), W1243 (DK+ Solids4Fun) and F4502-N16 (SFB FOXSI).

Open Access This article is distributed under the terms of the Creative Commons Attribution 4.0 International License (http://creativeco mmons.org/licenses/by/4.0/), which permits unrestricted use, distribution, and reproduction in any medium, provided you give appropriate credit to the original author(s) and the source, provide a link to the Creative Commons license, and indicate if changes were made.

\section{References}

1. Somorjai GA, Rupprechter G (1998) The flexible surface. J Chem Educ 75(2):161-176

2. Rupprechter G (2007) Sum frequency generation and polarization-modulation infrared reflection absorption spectroscopy of functioning model catalysts from ultrahigh vacuum to ambient pressure. In: Gates B, Knözinger H (eds) Advances in catalysis, vol 51, 1st edn. Academic Press, Cambridge, pp 133-263

3. Rupprechter G (2016) Surface science approach to heterogeneous catalysis. In: Wandelt K (ed) Surface and interface science: solidgas interfaces I, vol 5. Wiley, Weinheim, pp 459-528

4. Somorjai GA, McCrea KR (2000) Sum frequency generation: surface vibrational spectroscopy studies of catalytic reactions on metal single-crystal surfaces. In: Gates B, Knözinger H (eds) Advances in catalysis, vol 45, 1st edn. Academic Press, Cambridge, pp 385-438

5. Somorjai GA, Rupprechter G (1999) Molecular studies of catalytic reactions on crystal surfaces at high pressures and high temperatures by infrared-visible sum frequency generation (SFG) surface vibrational spectroscopy. J Phys Chem B 103(10):1623-1638

6. Somorjai GA, Su XC, McCrea KR, Rider KB (1999) Molecular surface studies of adsorption and catalytic reaction on crystal ( $\mathrm{Pt}, \mathrm{Rh}$ ) surfaces under high pressure conditions (atmospheres) using sum frequency generation (SFG) - surface vibrational spectroscopy and scanning tunneling microscopy (STM). Top Catal 8(1-2):23-34

7. Kung KY, Chen P, Wei F, Rupprechter G, Shen YR, Somorjai GA (2001) Ultrahigh vacuum high-pressure reaction system for 2-infrared 1-visible sum frequency generation studies. Rev Sci Instrum 72(3):1806-1809

8. Yang MC, Chou KC, Somorjai GA (2004) The structures and reactions of linear and cyclic $\mathrm{C}_{6}$ hydrocarbons adsorbed on the $\operatorname{Pt}(111)$ crystal surface by sum frequency generation vibrational spectroscopy pressure, temperature, and $\mathrm{H}_{2}$ coadsorption effects. J Phys Chem B 108(38):14766-14779

9. Yang M, Somorjai GA (2004) Unusual hydrogen effect in olefin dehydrogenation: 1-methylcyclohexene dehydrogenation initiated by excess hydrogen over $\mathrm{Pt}(111)$ surfaces, a combined sum frequency generation spectroscopy and kinetic study. J Phys Chem B 108(14):4405-4410

10. Yang M, Somorjai GA (2004) Adsorption and reactions of $\mathrm{C}_{6}$ hydrocarbons at high pressures on $\mathrm{Pt}(111)$ single-crystal surfaces studied by sum frequency generation vibrational spectroscopy: mechanisms of isomerization and dehydrocyclization of n-hexane. J Am Chem Soc 126(24):7698-7708

11. Thompson CM, Carl LM, Somorjai GA (2013) Sum frequency generation study of the interfacial layer in liquid-phase heterogeneously catalyzed oxidation of 2-propanol on platinum: effect of the concentrations of water and 2-propanol at the interface. J Phys Chem C 117(49):26077-26083

12. Sapi A, Liu FD, Cai XJ, Thompson CM, Wang HL, An KJ, Krier JM, Somorjai GA (2014) Comparing the catalytic oxidation of ethanol at the solid-gas and solid liquid interfaces over sizecontrolled Pt nanoparticles: striking differences in kinetics and mechanism. Nano Lett 14(11):6727-6730

13. Zhu ZW, Barroo C, Lichtenstein L, Eren B, Wu CH, Mao BH, de Bocarme TV, Liu Z, Kruse N, Salmeron M, Somorjai GA (2014) Influence of step geometry on the reconstruction of stepped platinum surfaces under coadsorption of ethylene and CO. J Phys Chem Lett 5(15):2626-2631

14. Baker LR, Kennedy G, Krier JM, Van Spronsen M, Onorato RM, Somorjai GA (2012) The role of an organic cap in nanoparticle catalysis: reversible restructuring of carbonaceous material controls catalytic activity of platinum nanoparticles for ethylene hydrogenation and methanol oxidation. Catal Lett 142(11):1286-1294

15. Krier JM, Michalak WD, Baker LR, An K, Komvopoulos K, Somorjai GA (2012) Sum frequency generation vibrational spectroscopy of colloidal platinum nanoparticle catalysts: disordering versus removal of organic capping. J Phys Chem C 116(33):17540-17546

16. Wang D, Penner S, Su DS, Rupprechter G, Hayek K, Schlögl R (2003) Silicide formation on a Pt/SiO${ }_{2}$ model catalyst studied by TEM, EELS, and EDXS. J Catal 219(2):434-441

17. Penner S, Wang D, Su DS, Rupprechter G, Podloucky R, Schlögl R, Hayek K (2003) Platinum nanocrystals supported by silica, alumina and ceria: metal-support interaction due to high-temperature reduction in hydrogen. Surf Sci 532:276-280

18. Hayek K, Goller H, Penner S, Rupprechter G, Zimmermann C (2004) Regular alumina-supported nanoparticles of iridium, rhodium and platinum under hydrogen reduction: structure, morphology and activity in the neopentane conversion. Catal Lett 92(1-2):1-9

19. Liu WT, Zhang LN, Shen YR (2006) Interfacial structures of methanol: water mixtures at a hydrophobic interface probed by sum-frequency vibrational spectroscopy. J Chem Phys 125(14): 144711

20. Feng RR, Guo Y, Wang HF (2014) Reorientation of the "free $\mathrm{OH}$ " group in the top-most layer of air/water interface of sodium fluoride aqueous solution probed with sum-frequency generation vibrational spectroscopy. J Chem Phys 141(18):18C507

21. Li X, Deng GH, Feng RJ, Lin K, Zhang Z, Bai Y, Lu Z, Guo Y (2016) Salt effect on molecular orientation at air/liquid methanol interface. Chin Chem Lett 27(4):535-539

22. Galletto P, Unterhalt H, Rupprechter G (2003) The molecular orientation of $\mathrm{CO}$ on $\mathrm{Pd}(111)$ : a polarization-dependent SFG study. Chem Phys Lett 367(5-6):785-790

23. Baldelli S, Markovic N, Ross P, Shen YR, Somorjai GA (1999) Sum frequency generation of CO on (111) and polycrystalline 
platinum electrode surfaces: evidence for SFG invisible surface CO. J Phys Chem B 103(42):8920-8925

24. Klünker C, Balden M, Lehwald S, Daum W (1996) CO stretching vibrations on $\operatorname{Pt}(111)$ and $\operatorname{Pt}(110)$ studied by sum-frequency generation. Surf Sci 360(1-3):104-111

25. Roiaz M, Pramhaas V, Li X, Rameshan C, Rupprechter G (2018) Atmospheric pressure reaction cell for operando sum frequency generation spectroscopy of ultrahigh vacuum grown model catalysts. Rev Sci Instr 89: 045104-1-045104-10

26. $\mathrm{Li} \mathrm{X}$ (2016) Investigation of molecular orientation and structure at air/electrolyte solution interface and phase transition of PNIPAM polymer at the air/ $\mathrm{H}_{2} \mathrm{O}$ interface using sum frequency generation vibrational spectroscopy. $\mathrm{PhD}$ Thesis, Institute of Chemistry, University of Chinese Academy of Sciences, Peking

27. Zhuang X, Miranda PB, Kim D, Shen YR (1999) Mapping molecular orientation and conformation at interfaces by surface nonlinear optics. Phys Rev B 59(19):12632-12640

28. Wang HF, Gan W, Lu R, Rao Y, Wu BH (2005) Quantitative spectral and orientational analysis in surface sum frequency generation vibrational spectroscopy (SFG-VS). Int Rev Phys Chem 24(2):191-256

29. Rupprechter G, Dellwig T, Unterhalt H, Freund HJ (2001) CO adsorption on $\mathrm{Ni}(100)$ and $\mathrm{Pt}(111)$ studied by infrared-visible sum frequency generation spectroscopy: design and application of an SFG-compatible UHV-high-pressure reaction cell. Top Catal 15(1):19-26

30. Hoffmann FM (1983) Infrared reflection-adsorption spectroscopy of adsorbed molecules. Surf Sci Rep 3:107-192

31. Rupprechter G, Dellwig T, Unterhalt H, Freund HJ (2001) Highpressure carbon monoxide adsorption on $\mathrm{Pt}(111)$ revisited: a sum frequency generation study. J Phys Chem B 105(18):3797-3802

32. Harle H, Mendel K, Metka U, Volpp HR, Willms L, Wolfrum J (1997) Temperature dependence (90-440 K) of the vibrational spectra of CO adsorbed on platinum(111) studied by sum-frequency generation. Chem Phys Lett 279(5-6):275-281

33. Agrawal VK, Trenary M (1991) An infrared study of NO adsorption at defect sites on Pt(111). Surf Sci 259(1-2):116-128

34. Miranda PB, Shen YR (1999) Liquid interfaces: a study by sum-frequency vibrational spectroscopy. J Phys Chem B 103(17):3292-3307

35. Reuttrobey JE, Doren DJ, Chabal YJ, Christman SB (1990) CO diffusion on $\operatorname{Pt}(111)$ with time-resolved infrared-pulsed molecular-beam methods - critical tests and analysis. J Chem Phys 93(12):9113-9129

36. Reuttrobey JE, Doren DJ, Chabal YJ, Christman SB (1988) Microscopic CO diffusion on a $\mathrm{Pt}(111)$ surface by time-resolved infrared-spectroscopy. Phys Rev Lett 61(24):2778-2781

37. Hayden BE, Kretzschmar K, Bradshaw AM, Greenler RG (1985) An infrared study of the adsorption of $\mathrm{CO}$ on a stepped platinum surface. Surf Sci 149(2-3):394-406

38. Greenler RG, Burch KD, Kretzschmar K, Klauser R, Bradshaw AM, Hayden BE (1985) Stepped single-crystal surfaces as models for small catalyst particles. Surf Sci 152-153(Part 1):338-345

39. Yuzawa T, Shioda T, Kubota J, Onda K, Wada A, Domen K, Hirose C (1998) Polarization characteristics from SFG spectra of clean and regulatively oxidized $\mathrm{Ni}(100)$ surfaces adsorbed by propionate and formate. Surf Sci 416(1-2):L1090-L1094

40. Rakic AD, Djurisic AB, Elazar JM, Majewski ML (1998) Optical properties of metallic films for vertical-cavity optoelectronic devices. Appl Opt 37(22):5271-5283

41. Wei X, Hong SC, Zhuang XW, Goto T, Shen YR (2000) Nonlinear optical studies of liquid crystal alignment on a rubbed polyvinyl alcohol surface. Phys Rev E 62(4):5160-5172

42. Oh-e M, Yokoyama H, Baldelli S (2004) Structure of the glycerol liquid/vapor interface studied by sum-frequency vibrational spectroscopy. Appl Phys Lett 84(24):4965-4967
43. Colles MJ, Griffith.Je (1972) Relative and absolute raman scattering cross-sections in liquids. J Chem Phys 56(7):3384-3391

44. Li ZG, Wang JX, Li YM, Xiong W (2016) Solving the "magic angle" challenge in determining molecular orientation heterogeneity at interfaces. J Phys Chem C 120(36):20239-20246

45. Scheijen FJE, Ferre DC, Niemantsverdriet JW (2009) Adsorption and dissociation of $\mathrm{CO}$ on body-centered cubic transition metals and alloys: effect of coverage and scaling relations. J Phys Chem C 113(25):11041-11049

46. McCrea K, Parker JS, Chen PL, Somorjai G (2001) Surface structure sensitivity of high-pressure $\mathrm{CO}$ dissociation on $\mathrm{Pt}(557)$, $\mathrm{Pt}(100)$ and $\mathrm{Pt}(111)$ using sum frequency generation surface vibrational spectroscopy. Surf Sci 494(3):238-250

47. Haghofer A, Sonström P, Fenske D, Föttinger K, Schwarz S, Bernardi J, Al-Shamery K, Bäumer M, Rupprechter G (2010) Colloidally prepared Pt nanowires versus impregnated Pt nanoparticles: comparison of adsorption and reaction properties. Langmuir 26(21):16330-16338

48. Schweizer E, Persson BNJ, Tüshaus M, Hoge D, Bradshaw AM (1989) The potential-energy surface, vibrational phase relaxation and the order-disorder transition in the adsorption system $\mathrm{Pt}(111)$ CO. Surf Sci 213(1):49-89

49. Ertl G, Neumann M, Streit KM (1977) Chemisorption of CO on Pt(111) surface. Surf Sci 64(2):393-410

50. Gan W, Wu BH, Chen H, Guo Y, Wang HF (2005) Accuracy and sensitivity of determining molecular orientation at interfaces using sum frequency generation vibrational spectroscopy. Chem Phys Lett 406(4-6):467-473

51. Li X, Feng RJ, Wang JJ, Zhang Z, Lu Z, Guo Y (2015) Role of refractive index in sum frequency generation intensity of salt solution interfaces. Chin Chem Lett 26(12):1542-1546

52. Demoulin O, Rupprechter G, Seunier I, Le Clef B, Navez M, Ruiz $P$ (2005) Investigation of parameters influencing the activation of a Pd/gamma-alumina catalyst during methane combustion. J Phys Chem B 109(43):20454-20462

53. de la Fuente OR, Borasio M, Galletto P, Rupprechter G, Freund HJ (2004) The influence of surface defects on methanol decomposition on Pd(111) studied by XPS and PM-IRAS. Surf Sci 566:740-745

54. Dellwig T, Rupprechter G, Unterhalt H, Freund HJ (2000) Bridging the pressure and materials gaps: high pressure sum frequency generation study on supported Pd nanoparticles. Phys Rev Lett 85(4):776-779

55. Rameshan C, Weilach C, Stadlmayr W, Penner S, Lorenz H, Hävecker M, Blume R, Rocha T, Teschner D, Knop-Gericke A, Schlögl R, Zemlyanov D, Memmel N, Rupprechter G, Klötzer B (2010) Steam reforming of methanol on PdZn near-surface alloys on Pd(111) and Pd foil studied by in-situ XPS, LEIS and PMIRAS. J Catal 276(1):101-113

56. Unterhalt H, Rupprechter G, Freund HJ (2002) Vibrational sum frequency spectroscopy on $\mathrm{Pd}(111)$ and supported Pd nanoparticles: $\mathrm{CO}$ adsorption from ultrahigh vacuum to atmospheric pressure. J Phys Chem B 106(2):356-367

57. Rupprechter G, Unterhalt H, Morkel M, Galletto P, Hu LJ, Freund HJ (2002) Sum frequency generation vibrational spectroscopy at solid-gas interfaces: $\mathrm{CO}$ adsorption on Pd model catalysts at ambient pressure. Surf Sci 502:109-122

58. Bourguignon B, Carrez S, Dragnea B, Dubost H (1998) Vibrational spectroscopy of imperfect $\mathrm{CO} / \mathrm{Pd}(111)$ surfaces obtained by adsorption between 150 and 230 K. Surf Sci 418(1):171-180

59. Morkel M, Unterhalt H, Salmeron M, Rupprechter G, Freund HJ (2003) SFG spectroscopy from $10^{-8}$ to 1000 mbar: less-ordered CO structures and coadsorption on Pd(111). Surf Sci 532:103-107

60. Rupprechter G, Unterhalt H, Morkel M, Galletto P, Dellwig T, Freund HJ (2003) Extending UHV studies to the mbar range: 
vibrational SFG spectroscopy of high-pressure CO adsorption on $\mathrm{Pt}(111)$ and $\mathrm{Pd}(111)$. Vacuum 71(1-2):83-87

61. Rupprechter G, Bandara A (2011) Sum frequency generation (SFG) spectroscopy. In: Friedbacher G, Bubert H (eds) Surface and thin film analysis: a compendium of principles, instrumentation, and applications, 2nd edn. Wiley, Weinheim, pp 407-435 